\title{
STECHKIN-MARCHAUD-TYPE INEQUALITIES OF WEIGHTED APPROXIMATION FOR BASKAKOV OPERATORS
}

\section{FENG GUO}

Abstract. In this paper, we introduce $\omega_{\varphi^{\lambda}}^{2}(f ; t)_{w, \lambda}$ and prove a generalization of weighed StechkinMarchaud-type inequality for Baskakov operators, from which the inverse result of Baskakov operators with $\omega_{\varphi^{\lambda}}^{2}(f ; t)_{w, \lambda}$ is obtained.

Mathematics subject classification (2010): 41A10, 41A16, 41A35.

Keywords and phrases: Baskakov operators, Stechkin-Marchaud-type inequality, weighted modulus of smoothness, $K$-functional.

\section{REFERENCES}

[1] V. A. Baskakov, An Example of a Sequence of Linear Positive Operators in the Spaces of Continuous Functions, Doki. Akad. Nauk. SSSR. 112 (1957), 249-251, MR. 20 \# 1153.

[2] M. Becker, A Global Approximation Theorem for Szasz-Mirakjan and Baskakov Operators in Polynomial Weighted Spaces, Indian. Univ. Math. J. 27 (1987), 127-142, MR\#12116.

[3] Z. Ditzian, On Global Inverse Theorems for Szasz and Baskakov Operators, Can. J. Math. 2 (1979), $255-263$.

[4] Z. DitZIAN AND K. Ivanov, Strong Converse Inequalities, J. Anal. Math. 61, 1 (1993), 61-111.

[5] Z. Ditzian, V. Totik, Moduli of Smoothness, Springer-Verlag, New York, 1987.

[6] V. Tотік, Strong Converse Inequalities, J. Approx. Theory 76 (1994), 369-375.

[7] J. J. WANG, Y. C. XUE, Stechkin-Marchaud-Type Inequalities of Weighted Approximation for Baskakov-Type Operators, J. Math. Research \& Exposition 24, 4 (2004), 710-714 (in Chinese).

[8] E. VAn. WiCKeren, Stechkin Marchaud-Type Inequalities in Connection with Bernstein Polynomials, Constr. Approx. 48, 2 (1986), 331-337.

[9] P. C. Xun, D. X. Zhou, Rate of Convergence for Baskakov Operators with Jacobi-Weights, Acta Mathematics Applicatae Sinica 18 (1995), 127-139 (in Chinese). 